\title{
Avoidance of interspecific mating in female Syrian hamsters is stronger toward familiar than toward unfamiliar heterospecific males
}

\author{
Javier delBarco-Trillo • Robert E. Johnston
}

Published online: 24 February 2011

(C) Psychonomic Society, Inc. 2011

\begin{abstract}
Adult Syrian hamster females (Mesocricetus auratus) learn to discriminate against familiar heterospecific males (Turkish hamster, M. brandti). We investigated whether females learn to avoid any heterospecific male after exposure to just one heterospecific male. We predicted that, after being exposed to one heterospecific male, a female would avoid mating not only with that familiar male but also with any unfamiliar heterospecific male. We exposed females to a heterospecific male across a wire-mesh barrier for 8 days and then paired the female with (a) that same heterospecific male or (b) an unfamiliar heterospecific male. Females exhibited lordosis faster and for a longer duration toward the unfamiliar than toward the familiar heterospecific male. However, females were similarly aggressive toward familiar and unfamiliar heterospecific males. Perhaps exposure to stimuli from several heterospecific males (a likely scenario in the wild) would result in females behaving similarly toward familiar and unfamiliar heterospecific males.
\end{abstract}

Keywords Familiarity · Interspecific mating · Species discrimination $\cdot$ Learning $\cdot$ Memory $\cdot$ Lordosis $\cdot$ Aggression

\section{Introduction}

There is strong selective pressure on females to avoid interspecific mating, because mating with heterospecific

J. delBarco-Trillo $\cdot$ R. E. Johnston

Department of Psychology, Cornell University,

Uris Hall,

Ithaca, NY 14853, USA

J. delBarco-Trillo $(\bowtie)$

Department of Evolutionary Anthropology, Duke University,

128 Biological Sciences Building, Box 90383, Durham,

NC 27708, USA

e-mail: delbarcotrillo@gmail.com males normally leads to a reduction in fitness (Gröning \& Hochkirch, 2008). This selective pressure has led females in many species to be unreceptive and/or aggressive toward heterospecific males (Andersson, 1994). This type of discriminative behavior against heterospecifics can be innately determined (Hebets, 2007), or it may require some type of learning during early development-for example, imprinting (Irwin \& Price, 1999; Servedio, Sæther, \& Sætre 2009; ten Cate \& Vos, 1999). Learning to discriminate between conspecific and heterospecific individuals during adulthood has been shown in male fruit flies and male guppies (Dukas, 2004, 2008; Magurran \& Ramnarine, 2004). In contrast, female guppies seem unable to learn to discriminate against heterospecific males (Haskins \& Haskins, 1949). Learning to avoid interspecific mating by females during adulthood has only been shown in Syrian hamster females, Mesocricetus auratus, toward Turkish hamster males, M. brandti (delBarco-Trillo \& Johnston, 2011; delBarco-Trillo, McPhee, \& Johnston 2010). In the first study on the topic of whether exposure to heterospecific males can affect the behavior of Syrian hamster females toward heterospecific males, we showed that 8 days of exposure to both a heterospecific male and a conspecific male reduces sexual receptivity toward the familiar heterospecific male, whereas the behavior of the female does not change toward the familiar conspecific male (delBarcoTrillo et al., 2010). Our second and last published study on the topic differed from our first one, in that during the exposure phase we used smaller arenas and females were only exposed to a heterospecific male. The results were comparable to those in delBarco-Trillo et al. (2010). Briefly, we showed that 4 days of exposure had consequences similar to those for 8 days of exposure, whereas shorter durations ( 1 or $4 \mathrm{~h}$ ) resulted in females still being sexually receptive toward the familiar heterospecific male (delBarco-Trillo \& Johnston, 2011). In the present study, 
using the same smaller arenas and 8-day exposure only to a heterospecific male, we investigated whether females behave similarly toward a familiar heterospecific male (i.e., the heterospecific male she was exposed to for 8 days) or an unfamiliar heterospecific male.

Previously, we reported that estrous female Syrian hamsters show a preference for the odors of conspecific males over those of heterospecific Turkish hamster males (delBarco-Trillo, Gulewicz, \& Johnston 2009; delBarcoTrillo, Gulewicz, Segal, McPhee, \& Johnston 2009). However, Syrian hamster females do not innately avoid mating with heterospecific males (delBarco-Trillo et al., 2009; delBarco-Trillo \& Johnston, 2010), despite the fact that mating between Turkish and Syrian hamsters does not produce viable offspring (Murphy, 1977; Todd, Nixon, Mulvaney, \& Connelly 1972); at present, these two species have nonoverlapping geographical distributions (Neumann, Michaux, Lebedev, Yigit, Colak, Ivanova 2006), but there may have been episodes of sympatry since the inception of both species (delBarco-Trillo \& Johnston, 2011). That is, when Syrian hamster females without any prior experience with heterospecific males are paired with a heterospecific male, all females are receptive to and do not show any sign of aggression toward heterospecific males (delBarco-Trillo et al., 2009; delBarco-Trillo \& Johnston, 2010). However, when females were exposed for 8 days to a heterospecific male across a wire-mesh partition, females were then aggressive and not sexually receptive when paired with the same heterospecific male (delBarco-Trillo \& Johnston, 2011; delBarco-Trillo et al., 2010). In these previous studies, we did not test females with an unfamiliar heterospecific male after they had been exposed for 8 days to another heterospecific male. A priori, there was no reason to expect that a female would behave differently toward familiar and unfamiliar heterospecific males. However, in a recent study, male Trinidadian guppies (Poecilia reticulata) were exposed for 14 days to female Skiffia bilineata (a distantly related species), and after this exposure, males reduced mating attempts toward familiar heterospecific females but persisted in attempting to mate with unfamiliar heterospecific females (Valero, Magurran, \& Garcia 2009). That is, males did not generalize the learned response for the familiar heterospecific female to unfamiliar heterospecific females (Valero et al., 2009).

Here we determined whether Syrian hamsters differ from Trinidadian guppies (Valero et al., 2009) by being able to generalize their learned response for a familiar heterospecific to unfamiliar heterospecifics. Whether female Syrian hamsters generalize their response to any heterospecific male may depend on the type of information about males used by the females (Johnston \& Peng, 2008). Female hamsters may use individual information of the familiar heterospecific male. If so, females may be more efficient at avoiding mating with that familiar male than with unfamiliar heterospecific males. Alternatively, females may use cues that are characteristic of all males of the other species. If so, once females are exposed to one heterospecific male, they should be as able to avoid mating with any heterospecific male as they will be with that familiar male.

We investigated whether females learn to avoid mating with any heterospecific male after they have been exposed to one particular heterospecific male. We exposed a female to a heterospecific male for 8 days and then paired the female with (a) that same heterospecific male or (b) an unfamiliar heterospecific male. We measured sexual receptivity and aggression toward the heterospecific male. Given that familiar and unfamiliar heterospecific males do not, a priori, hold different values for a female, we predicted that receptive and aggressive behaviors by the subject females would be similar toward the familiar and the unfamiliar heterospecific males.

\section{Method}

\section{Animals}

All animals were born and raised in captivity at Cornell University, Ithaca, NY. Hamsters were weaned at 30 days of age and housed individually in solid-bottom polycarbonate cages $(45 \times 24 \times 14.5 \mathrm{~cm})$ with Sani-chip bedding material and constant access to water and food (Prolab 1000, Agway, Syracuse, NY). Turkish and Syrian hamsters were maintained in separate rooms with independent air intakes and exhausts. The Turkish hamsters were maintained on a 16:8 light-dark schedule with lights off between 10:00 and 18:00 h (Eastern Standard Time). The Syrian hamsters were maintained on a 14:10 light-dark schedule with lights off between 09:00 and 19:00 h. The different light cycles were necessary for husbandry reasons (Turkish hamsters seem to require longer light cycles to reproduce successfully in captivity). Experiments were run between 10:00 and 13:00 h. We used dim and indirect light in order to allow for videotaping and observations. The video camera was located approximately $1 \mathrm{~m}$ from the cage in which the interactions took place. The female test subjects were not siblings.

To determine that a female was in estrus on a specific day, a conspecific male hamster was placed inside the female's home cage. If lordosis occurred within $15 \mathrm{~s}$, the female was considered in estrus (no mounting occurred during estrous testing). Lordosis is a stereotypical position in Mesocricetus spp. (and other rodents) in which the female arches her back (down in the center), spreads her legs, raises her tail, and remains immobile from seconds to 
minutes (Carter, 1985). A male can only mate if a female is in lordosis, which is indicative of sexual receptivity (Floody \& Pfaff, 1977). Consequently, if a female shows lordosis toward conspecific males but not toward heterospecific males, this would be an effective response to avoid interspecific mating. If no lordosis was observed, the female was retested on the following days (up to 3 days), until lordosis occurred. Given the regular 4-day estrous cycle in Syrian hamsters (Lisk, 1985), once the day of estrus was determined, we could always determine the subsequent estrous days. In this study, females were always tested 4 days after determining the timing of their estrous cycles (i.e., during their next estrous day). All females underwent estrous checking, so this experience should have had no effect on the results.

\section{Experimental design}

We used 10 arenas $(50 \times 38.1 \times 21.5 \mathrm{~cm}$; Allentown, Model PCT4SHT) divided into two similarly sized sections $(24.85 \times 38.1 \times 21.5 \mathrm{~cm})$ by a wire-mesh partition with $1-\mathrm{cm}^{2}$ openings. This type of barrier is sufficient for hamsters to learn the individual identity of other conspecifics (Johnston \& Peng, 2008). We started a test by placing one Turkish hamster male in one of the two sections, and then an estrous, Syrian hamster female in the other section. The female could perceive and interact in a limited manner with the male across the wire-mesh partition. We used soiled bedding from each animal's home cage as substrate for its respective section of the arena. Each animal had constant access to its own water and food. The animals lived undisturbed in this arena for 8 days. The arenas were placed in a room separate from the colony and testing rooms; the light cycle in this room was a 14:10 light-dark schedule, with lights off between 09:00 and 19:00 h. Each arena was placed in a different rack and covered with a filtered top in order to avoid transfer of visual or olfactory (but not necessarily auditory) information between arenas. After the 8 days of exposure, the female and the male in each arena were transferred to individual cages lined with clean bedding. Females were assigned to one of two groups. In the familiar-heterospecific group $(n=10)$, the female was tested with the same heterospecific male that she had lived next to for 8 days. In the unfamiliar-heterospecific group $(n=10)$, the female was tested with an unfamiliar heterospecific male; in this group, we set up pairs of arenas so that on the testing day we paired the female in the first arena with the heterospecific male in the second arena, and the female in the second arena with the heterospecific male in the first. In all tests, the pairing trial started $10 \mathrm{~min}$ after the animals had been transferred to their individual cages. The female and the male to be paired were moved in their separate cages to the testing room. The male was transferred to the female cage in a clean, 500-ml plastic beaker. The trial lasted $5 \mathrm{~min}$. We did not interfere with the behavior of animals during these trials. We videotaped all trials, and determined whether the female sniffed the male before displaying lordosis or aggressive behavior (such initial sniffing occurred in all trials). A researcher blind to the treatments scored the recorded videos to determine the latency to lordosis, the duration of lordosis, the latency to aggression, and the duration of aggression. We considered any attempted biting or tumbling fights as signs of aggression. If a female did not show lordosis or aggression during a test, we scored the latency and duration for those behaviors as 300 and $0 \mathrm{~s}$, respectively. If the female was aggressive and not receptive to the heterospecific male, the female was immediately paired with an unfamiliar conspecific male for $5 \mathrm{~min}$ to determine that the female was indeed in estrus. All females tested were in estrus. Such estrus-checking trials were not used for any statistical analyses.

\section{Statistics}

We used independent $t$ tests to determine whether any of the variables (latency to lordosis, duration of lordosis, latency to aggression, or duration of aggression) differed between the two groups (familiar-heterospecific and unfamiliar-heterospecific).

We tested the assumption of normality using the Kolmogorov-Smirnov test. We used inverse transformation to normalize the data for latency to lordosis, and Levene's test for equality of variances. Equal variances were not assumed for latency to lordosis and duration of lordosis, and we report corrected degrees of freedom for these two variables. The values in the Results are shown as means \pm SEMs. We used SPSS 14 for Windows for all statistical analyses.

\section{Results}

The latency to lordosis was significantly longer in the familiar-heterospecific group $(225.3 \pm 38.09 \mathrm{~s})$ than in the unfamiliar-heterospecific group $(114.8 \pm 40.8 \mathrm{~s})[t(10.64)=$ $-2.22, p=.049$; see Fig. 1a]. The duration of lordosis was significantly shorter in the familiar-heterospecific group $(40.3 \pm 23.98 \mathrm{~s})$ than in the unfamiliar-heterospecific group $(125.76 \pm 32.59$ s) $[t(16.54)=-2.11, p=.050$; Fig. 1b]. Copulation occurred in $10 \%$ of the 5 -min trials (1 of 10 ) in the familiar-heterospecific group, but in $60 \%$ of the trials (6 of 10) in the unfamiliar-heterospecific group. No lordosis occurred in $70 \%$ of the trials ( 7 of 10 ) in the familiarheterospecific group, as compared to $30 \%$ of trials (3 of 10) in the unfamiliar-heterospecific group. These results indicate that female Syrian hamsters were more sexually 
a

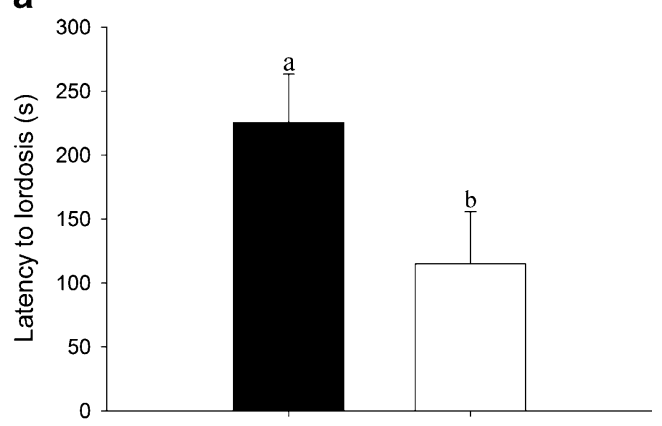

b

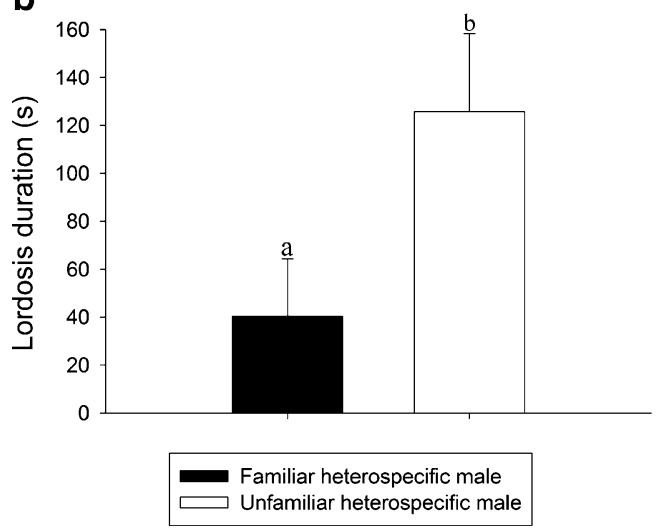

Fig. 1 Latency to lordosis (a) and lordosis duration (b) by female Syrian hamsters (Mesocricetus auratus) paired with a familiar heterospecific male (Turkish hamster, M. brandti; black bars) or an unfamiliar heterospecific male (white bars). Different letters denote a statistically significant difference. Values represent means $\pm S E M \mathrm{~s}$

receptive toward the unfamiliar than toward the familiar heterospecific male. In all of the trials in which the female did not show lordosis toward the heterospecific male, the female was highly receptive when she was immediately paired with an unfamiliar conspecific male.

There were no significant differences between the familiar- and unfamiliar-heterospecific groups in latencies to aggression $[t(18)=-0.71, p=.49$; Fig. 2a] or in the duration of aggression $[t(18)=0.54, p=.59$; Fig. 2b]. Aggression occurred in $90 \%$ of the 5-min trials (9 of 10) in the familiar-heterospecific group and in $70 \%$ of the trials (7 of 10) in the unfamiliar-heterospecific group.

In most trials, females that were aggressive toward a heterospecific male did not show lordosis, and vice versa. Aggression and lordosis in the same trial occurred in $20 \%$ of the trials ( 2 of 10) in the familiar-heterospecific group and in $40 \%$ of the trials (4 of 10) in the unfamiliar-heterospecific group.

\section{Discussion}

We investigated whether females learn to avoid mating with an unfamiliar heterospecific male after they have been exposed to a different heterospecific male. The results show that female Syrian hamsters that have been exposed to a heterospecific male for 8 days avoid mating with that familiar heterospecific male more than they do with an unfamiliar heterospecific male.

We had predicted that whether female Syrian hamsters generalize their response to any heterospecific male should depend on the type of information that females learn during their exposure to a given heterospecific male. If females learn a type of information that can differ significantly from individual to individual, they may indeed be more efficient at avoiding mating with the familiar heterospecific male than with unfamiliar heterospecific males (Pfennig \& Pfennig, 2009). Syrian hamsters can use odors from different body sources to develop multicomponent representations of other conspecifics, and it is feasible that they can also do so for individuals of a closely related speciesfor example, Turkish hamsters (Johnston \& Peng, 2008). Alternatively, females may use cues that are characteristic of all heterospecific males (Dalesman \& Rundle, 2010). If so, once females are exposed to a heterospecific male, they should be able to avoid mating with any heterospecific male. Our results suggest that females exposed to a heterospecific male learn individual characteristics of that
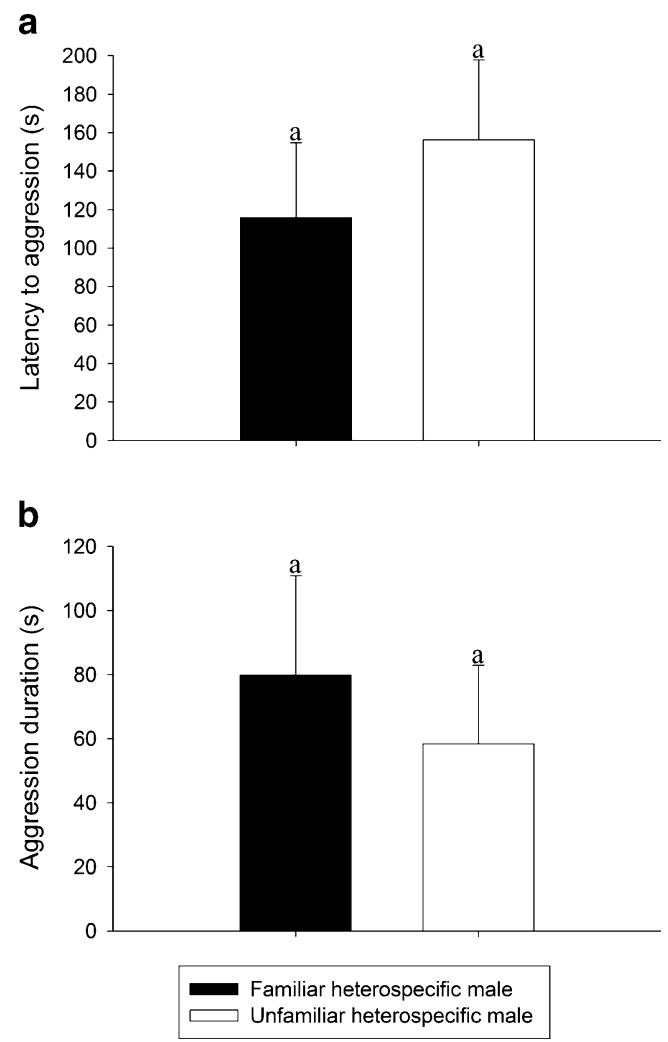

Fig. 2 Latency to aggression (a) and aggression duration (b) by female Syrian hamsters (Mesocricetus auratus) paired with a familiar heterospecific male (Turkish hamster, M. brandti; black bars) or an unfamiliar heterospecific male (white bars). Similar letters denote the lack of a statistically significant difference. Values represent means $\pm S E M \mathrm{~s}$ 
male and not species-broad characteristics expressed by all heterospecific males.

In another study, we have shown that if a female is exposed to a heterospecific male for 8 days, then isolated from that heterospecific male for 8 days, and finally paired with that heterospecific male, such a female is more sexually receptive and less aggressive than are females that were isolated from the familiar heterospecific male for only 10 min between the initial 8-day exposure and pairing the female with that male (delBarco-Trillo \& Johnston, 2011). In that study, we suggested two possible explanations for this finding. First, females may form a short-term memory of the heterospecific male, and that memory may be lost within 8 days in the absence of exposure to that male. Second, exposure to a heterospecific male may change the physiological state of the female, for example by changes in hormone concentrations in blood or neuroreceptors in the brain. After 8 days without contact with heterospecifics, the physiological state of the female may return to baseline levels, and thus females may start behaving like naïve females - that is, not behaving differently toward conspecific and heterospecific males. The data in the present study do not support the second, physiological explanation, because if exposure to a heterospecific male changes the physiological state of a female so that the female becomes able to avoid mating with heterospecific males, she should show this avoidance toward any heterospecific male. The fact that females avoided the familiar more than the unfamiliar heterospecific male suggests that females did form a memory of the familiar male.

The conditions used in this study and in two previous studies (delBarco-Trillo \& Johnston, 2011; delBarco-Trillo et al., 2010) prevented the male from engaging in sexual behavior with the female throughout the 8 days of their interaction. The female might thus have treated that male as an unsuitable mate, independently of whether that male is a conspecific or a heterospecific. However, in a previous study (delBarco-Trillo et al., 2010) in which females were exposed to a conspecific male or two conspecific males for 8 days behind wire-mesh partitions, those females were still highly sexually receptive to those familiar conspecific males. Consequently, the lack of copulatory behavior per se during the 8 days of separated cohabitation is unlikely to explain the unreceptive behavior of females toward familiar heterospecific males.

Our results are similar to those shown in male Poecilia guppies exposed to females of a distantly related species (Valero et al., 2009), in that avoidance of mating toward familiar heterospecific individuals was greater than avoidance toward unfamiliar individuals. These results are surprising, since there is no obvious reason for females to avoid some heterospecific males more than others. That is, the ability to discriminate between different conspecifics is valuable in mate choice and nepotism; however, discriminating between and behaving differently toward familiar and unfamiliar heterospecifics does not seem to provide any significant advantage. It could be argued that our surprising results are due to the fact that we exposed females to only one heterospecific male. In the wild, if two given species are sympatric, females are likely to be exposed to more than one heterospecific male. It is possible that, after being exposed to several heterospecific males, females would combine information from those different males to develop a general representation of any heterospecific male, resulting in females then behaving similarly toward familiar and unfamiliar heterospecific males.

We did not find statistically significant differences in the aggressive behavior of females toward familiar and unfamiliar heterospecific males. We previously showed that sexual and aggressive behaviors of female Syrian hamsters toward heterospecific males can be dissociated: A short exposure to a heterospecific male was sufficient to elicit aggression in females, but much longer exposures were required to significantly reduce the duration of lordosis toward the heterospecific male (delBarco-Trillo \& Johnston, 2011). It remains to be investigated whether different physiological and/or cognitive mechanisms are involved in the aggressive and sexual responses toward heterospecific individuals.

Reproductive interference involves any negative sexual interaction between species (Crowder, Sitvarin, \& Carrière 2010; Kishi, Nishida, \& Tsubaki 2009). The present study contributes to our understanding of reproductive interference, especially to the mostly unstudied topic of how females may decrease the fitness-reducing effects of reproductive interference. Instead of innate responses to minimize reproductive interference, females in some species may use information from their interactions with heterospecific individuals to adjust their behavior, and thus reduce the negative effects of reproductive interference. The present study suggests that limited information-for example, interactions with only one heterospecific malemay be insufficient to generate a successful response against any further heterospecifics.

\section{References}

Andersson, M. (1994). Sexual selection. Princeton: Princeton University Press.

Carter, C. S. (1985). Female sexual behavior. In H. I. Siegel (Ed.), The Hamster: Reproduction and behavior (pp. 173-189). New York: Plenum Press.

Crowder, D. W., Sitvarin, M. I., \& Carrière, Y. (2010). Plasticity in mating behaviour drives asymmetric reproductive interference 
in whiteflies. Animal Behaviour, 79, 579-587. doi:10.1016/j. anbehav.2009.11.025.

Dalesman, S., \& Rundle, S. D. (2010). Cohabitation enhances the avoidance response to heterospecific alarm cues in a freshwater snail. Animal Behaviour, 79, 173-177.

delBarco-Trillo, J., Gulewicz, K., \& Johnston, R. E. (2009). Medial amygdala involvement in discrimination of same-species and closely-related-species male stimuli in estrous female Mesocricetus hamsters. Behavioral Neuroscience, 123, 758-763. doi:10.1037/ a0016439.

delBarco-Trillo, J., Gulewicz, K., Segal, A., McPhee, M. E., \& Johnston, R. E. (2009). Can captivity lead to inter-species mating in two Mesocricetus hamster species? Journal of Zoology, 278, 308-312. doi:10.1111/j.1469-7998.2009.00577.x.

delBarco-Trillo, J., \& Johnston, R. E. (2010). Fluoxetine does not prevent interspecific mating between two hamster species. Physiology \& Behavior, 99, 684-686. doi:10.1016/j.physbeh. 2010.02.003.

delBarco-Trillo, J., \& Johnston, R. E. (2011). Adult female hamsters require long and sustained exposures to heterospecific males to avoid interspecific mating. Evolutionary Ecology, 25, 391-401. doi:10.1007/s10682-010-9429-7.

delBarco-Trillo, J., McPhee, M. E., \& Johnston, R. E. (2010). Adult female hamsters avoid interspecific mating after exposure to heterospecific males. Behavioral Ecology and Sociobiology, 64, 1247-1253. doi:10.1007/s00265-010-0939-7.

Dukas, R. (2004). Male fruit flies learn to avoid interspecific courtship. Behavioral Ecology, 15, 695-698. doi:10.1093/ beheco/arh068.

Dukas, R. (2008). Learning decreases heterospecific courtship and mating in fruit flies. Biology Letters, 4, 645-647. doi:10.1098/ rsbl.2008.0437.

Floody, O. R., \& Pfaff, D. W. (1977). Aggressive behavior in female hamsters: The hormonal basis for fluctuations in female aggressiveness correlated with estrous state. Journal of Comparative and Physiological Psychology, 91, 443-464.

Gröning, J., \& Hochkirch, A. (2008). Reproductive interference between animal species. The Quarterly Review of Biology, 83, 257-282. doi:10.1086/590510.

Haskins, C. P., \& Haskins, E. F. (1949). The role of sexual selection as an isolating mechanism in three species of Poeciliid fishes. Evolution, 3, 160-169.

Hebets, E. A. (2007). Subadult female experience does not influence species recognition in the wolf spider Schizocosa uetzi Stratton 1997. Journal of Arachnology, 35, 1-10.

Irwin, D. E., \& Price, T. (1999). Sexual imprinting, learning and speciation. Heredity, 82, 347-354. doi:10.1038/sj.hdy.6885270.

Johnston, R. E., \& Peng, A. (2008). Memory for individuals: Hamsters (Mesocricetus auratus) require contact to develop multicomponent representations (concepts) of others. Journal of Comparative Psychology, 122, 121-131. doi:10.1037/07357036.122.2.121

Kishi, S., Nishida, T., \& Tsubaki, Y. (2009). Reproductive interference determines persistence and exclusion in species interactions. The Journal of Animal Ecology, 78, 1043-1049. doi:10.1111/j.13652656.2009.01560.x.

Lisk, R. D. (1985). The estrous cycle. In H. I. Siegel (Ed.), The Hamster: Reproduction and behavior (pp. 23-51). New York: Plenum Press.

Magurran, A. E., \& Ramnarine, I. W. (2004). Learned mate recognition and reproductive isolation in guppies. Animal Behaviour, 67, 1077-1082. doi:10.1016/j.anbehav.2003.10.010.

Murphy, M. R. (1977). Intraspecific sexual preferences of female hamsters. Journal of Comparative and Physiological Psychology, 91, 1337-1346.

Neumann, K., Michaux, J., Lebedev, V., Yigit, N., Colak, E., Ivanova, N., et al. (2006). Molecular phylogeny of the Cricetinae subfamily based on the mitochondrial cytochrome $\mathrm{b}$ and $12 \mathrm{~S}$ rRNA genes and the nuclear vWF gene. Molecular Phylogenetics and Evolution, 39, 135-148. doi:10.1016/j.ympev.2006.01.010.

Pfennig, K. S., \& Pfennig, D. W. (2009). Character displacement: Ecological and reproductive responses to a common evolutionary problem. The Quarterly Review of Biology, 84, 253-276.

Servedio, M., Sæther, S., \& Sætre, G.-P. (2009). Reinforcement and learning. Evolutionary Ecology, 23, 109-123. doi:10.1007/ s10682-007-9188-2.

ten Cate, C., \& Vos, D. (1999). Sexual imprinting and evolutionary processes in birds. Advances in the Study of Behavior, 28, 1-31. doi:10.1016/S0065-3454(08)60214-4.

Todd, N. B., Nixon, C. W., Mulvaney, D. A., \& Connelly, M. E. (1972). Karyotypes of Mesocricetus brandti and hybridization within the genus. The Journal of Heredity, 63, 73-77.

Valero, A., Magurran, A. E., \& Garcia, C. M. (2009). Guppy males distinguish between familiar and unfamiliar females of a distantly related species. Animal Behaviour, 78, 441-445. doi:10.1016/j. anbehav.2009.05.018.

\section{Author Note}

This work was supported by National Institute of Mental Health Grant NIMH 5 R01 MHO58001-08 to R.E.J. The experiments here described comply with the current laws of the USA. All research was conducted with approval from Cornell University's Institutional Animal Care and Use Committee (protocol \#1993-0120). The authors declare that they have no conflict of interest. 